\title{
Implicit Surface Reconstruction with an Analogy of Polar Field Model
}

\author{
Yuxu Lin ${ }^{1}$, Chun Chen ${ }^{1}$, Mingli Song ${ }^{1, \star}$, Jiajun $\mathrm{Bu}^{1}$, and Zicheng $\mathrm{Liu}^{2}$ \\ ${ }^{1}$ College of Computer Science, Zhejiang University \\ linyuxu@zju.edu.cn, \\ chenc@zju.edu.cn, \\ brooksong@ieee.org, \\ bjj@zju.edu.cn \\ 2 Microsoft Research Redmond \\ zliu@microsoft.com
}

\begin{abstract}
Implicit surface reconstruction has been a challenging work for decades. In this paper, motivated by the concept of classic physical polar field model and off-set points strategy, we present a new approach, called Field Fitting. In this approach, we express a 3D surface as an equipotential surface of scalar polar field which is produced by a number of paired field generating primitives, then a surface reconstruction process is cast as a primitives localization process, and finally, we solve this problem with a greedy method. Experimental results demonstrate that the proposed method outperforms the previous by providing better surface reconstruction results.
\end{abstract}

Keywords: Surface fitting, reconstruction, polar field, implicit surface, Field Fitting.

\section{Introduction}

Implicit Surface [1] is widely used for a compact representation of surface, which can produce higher resolution surfaces by sampling on predefined implicit function. Typically, an implicit surface reconstruction problem can be described as: given a point cloud along the surface, to find an implicit function $F(\boldsymbol{x})$ whose zero-sets approximate the original surface as accurate as possible. However, a 3D surface usually exhibits not only the smooth plane, but also the subtle and sharp details caused by corners, deformations etc. Due to such complexity of 3D surface, robust and accurate surface fitting is still a challenging work for computer graphics and geometric modeling researchers.

There have been a lot of approaches in surface fitting in the past decades. It is virtually impossible to enumerate all of them. The book written by Dierckx [2] and the review by Floater et al. 3] provided excellent surveys. Here we would like to review some representative implicit surface reconstruction approaches presented very recently.

\footnotetext{
* Corresponding author.
}

T. Wada, F. Huang, and S. Lin (Eds.): PSIVT 2009, LNCS 5414, pp. $4364448,2009$.
(c) Springer-Verlag Berlin Heidelberg 2009 
The first group is global fitting method, which commonly define one global function to express the surface, such as polynomial surface [4 and radius basis functions(RBFs) [5]6. The most popular global fitting method is RBFs which have been proved extremely effective to construct implicit surface from points and widely used, however, the solving process of ideal RBFs involve a large solution matrix which is rather time consuming, a practical solution (FastRBF) on large data sets involves adaptive RBF center reduction and fast multipole $\operatorname{method}[5]$.

The second group is local fitting method, which generates implicit function for each local patches. In [7, the implicit function was defined as the signed distance to the tangent plane of the closest point, and in [89], a moving least squares (MLS) projection was performed to find local function, and in [10, a technique named Multi-level Partition of Unity Implicits(MPU) was developed to reconstruct implicit surface as the blending of local implicit functions, MPU performs efficient on large scale data sets.

Very recently, a novel "Poisson Surface Reconstruction" was introduced in [1] which treated the surface reconstruction process as a spatial Poisson problem, and the experiments showed that it was also an effective way.

In this paper, inspired by the concept of classic physical polar field model and off-set points strategy in 3L algorithm [4, we propose a new implicit surface reconstruction approach called Field Fitting. By making an analogy between the fitting surface and the equipotential surface yielded by paired primitives, the implicit surface fitting is treated as a process of estimating the paired primitives' distribution. Then, the implicit surface fitting is formulated into an energy minimization problem. A greedy strategy is used to solve this problem iteratively. Moreover, multi-scaling strategy is adopted to overcome the unwanted holes on the surface. The experimental results demonstrate that the proposed Field Fitting approach outperforms previous ones by providing better surface fitting result on different 3D models.

This paper introduces the following key-contributions:

i) A novel implicit surface fitting approach is presented to carry out the reconstruction in a simple and effective way by analogy of a physical polar field model.

ii) The implicit surface fitting is formulated into an energy minimization problem. And,

iii) a greedy method is introduced to solve the energy minimization problem, which is more effective than conventional solving strategy.

The paper is organized as follows. Firstly, in section 2, we briefly describe the original physical polar field model and the off-set points' strategy in implicit surface reconstruction. Then, we describe our surface reconstruction approach in section 3. In section 4 and section 5, we explain how to extract isosurface and evaluate the proposed approach respectively. Finally, we conclude in section 6 . 

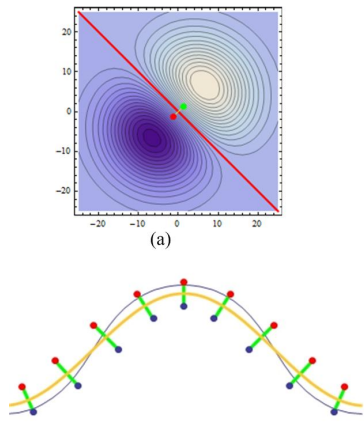

(d)

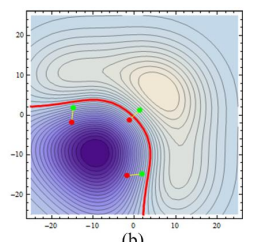

(b)

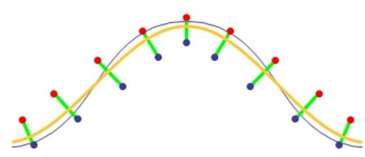

(e)

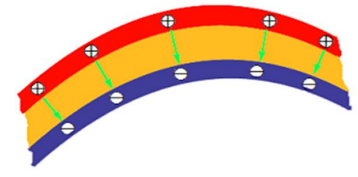

(c)

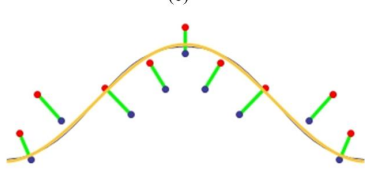

(f)

Fig. 1. From top to bottom and from left to right, (a): the equipotential curves yielded by a paired primitives with opposite polarity, the red line denote the zero potential curve, green point denote positive primitive and red point denote negative primitive; (b): the equipotential curves yielded by three paired primitives with opposite polarity, the zero potential curve does not go through the middle of all three paired primitives;(c): a typical profile of The Parallel-Plate Capacitor model, the red and blue stripe are paralleled conductor plates which carry equal quantity but opposite positive/negative primitives, and the yellow part in the middle is the insulator which separates the two paralleled conductors; (d): the zero potential curve(blue curve) for Parallel-Plate Capacitor; (e): the zero potential curve after adjusting positive/negative primitives for 10 times with Quasi-Newton method; (f): the zero potential curve after 120 rounds positive/negative primitives adjustments

\section{Preliminary}

\subsection{Physical Polar Field Model}

In physics world, there are two types of fields, the first type is nonpolar fields, such as gravity field and temperature field, the second type is polar fields, such as magnetic field and electric field which can be yield by two types of primitives with opposite polarity. For a field, given the location of primitives and the potential function, there will be an equipotential surface in field. Such characteristic motivates us greatly for a better fitting of implicit surfaces. If we regard the surface as an equipotential surface to be reconstructed, and the fitting points as the sampling of the surface, a surface reconstruction problem can be transformed to a procedure of estimating the primitives' distribution.

Considering a pair of primitives with same strength but opposite polar (we name them as positive and negative primitive), they set up field across the space and their corresponding equipotential curves in a plane are shown in Figure 1(a), the red line denote the zero potential curve, the green point denote the positive primitive and the red point denote the negative primitive, for a single paired primitives, the zero potential curve go through the middle of two primitives. In Figure 1 (b), the equipotential curves governed by three paired primitives are plotted, and it is noticeable that the zero potential curve does not go through 
the middle of all three paired primitives. The potential function of a single positive/negative primitives is described as:

$$
w^{+}(\boldsymbol{r})=\psi(|r|), \quad w^{-}(\boldsymbol{r})=-\psi(|r|)
$$

where $|\boldsymbol{r}|$ is the $3 \mathrm{D}$ distance from the positive/negative primitive. $\psi$ is a monotonic decreasing function according to $|r|$.

Making an extension of the simple polar field model with a few positive/ negative primitives mentioned above, a model named Parallel-Plate Capacitor with a pair of paralleled conductors (red and blue part in Figure 1 (c)) (only a patch is plotted) has plenty of primitives (Figure1(c)) which have equal strength but opposite polarity (positive/negative) primitives. Besides, there is an insulator in the middle of the capacitor (yellow part in Figure 1 (c), it intend to be infinite thin, and we thicken it for easily observation) which separates the two paralleled conductors. We plot the zero potential curve for Parallel-Plate Capacitor (blue line in Figure1(d)), it is noticeable that the zero potential curve (blue line) is close to the insulator(yellow line), by adjusting the position of primitives (with Quasi-Newton method [12], discussed in section 3) several times (10 times in Figure 1 (e) and 120 times in Figure 1 (f)), the zero potential curve can fit the insulator. In other words, by adjusting the position of positive/negative primitives, the insulator can be defined as a zero set of the following implicit function:

$$
f(\boldsymbol{p})=\sum_{i=1}^{n} w_{i}^{+}\left(\boldsymbol{p}-\boldsymbol{L}_{\boldsymbol{i}}^{+}\right)+\sum_{i=1}^{n} w_{i}^{-}\left(\boldsymbol{p}-\boldsymbol{L}_{\boldsymbol{i}}^{-}\right)
$$

where $n$ stands for the number of positive/negative primitives, $\boldsymbol{L}_{\boldsymbol{i}}^{+} / \boldsymbol{L}_{\boldsymbol{i}}^{-}$is the position of $i_{\text {_th }}$ positive/negative primitives. $f(\boldsymbol{p})$ denotes the potential yield by all the primitives at point $\boldsymbol{p}$.

\subsection{Off-Set Points Strategy}

In 3L algorithm [4], given a points set $P=\left\{\boldsymbol{p}_{\boldsymbol{i}}\right\}$ and its corresponding normal set $N=\left\{\boldsymbol{n}_{\boldsymbol{i}}\right\}$ on a surface, we can approximate the surface with an implicit function $f$ in terms of an off-set points strategy. $f$ satisfies $f\left(\boldsymbol{p}_{\boldsymbol{i}}\right)=0$. In addition, $f\left(\boldsymbol{p}_{\boldsymbol{i}^{+}}\right)>0$ and $f\left(\boldsymbol{p}_{\boldsymbol{i}^{-}}\right)<0$ where $\boldsymbol{p}_{\boldsymbol{i}}^{+}=\boldsymbol{p}_{i}+\lambda \cdot \boldsymbol{n}_{\boldsymbol{i}}, \boldsymbol{p}_{\boldsymbol{i}}^{-}=\boldsymbol{p}_{i}-\lambda \cdot \boldsymbol{n}_{\boldsymbol{i}}, \lambda>0$, it means that given points which are in the zero-set of the implicit surface, we can infer that the implicit function value of a point will be positive when it moves forwards along the normal and negative when it moves backwards along the normal.

This property is usually utilized for constructing implicit surface as follows:

$$
\begin{aligned}
& f\left(\boldsymbol{p}_{\boldsymbol{i}}^{-}\right)=-c \\
& f\left(\boldsymbol{p}_{\boldsymbol{i}}\right)=0 \quad \text { where } c>0 \\
& f\left(\boldsymbol{p}_{\boldsymbol{i}}^{+}\right)=c
\end{aligned}
$$

The extra equations $f\left(\boldsymbol{p}_{\boldsymbol{i}}^{-}\right)=-c$ and $f\left(\boldsymbol{p}_{\boldsymbol{i}}^{+}\right)=c$ are used to enhance the stability of reconstruction process. And in our Field Fitting approach, we utilize the offset points to generate the positive and negative primitives (in analogy of $\boldsymbol{p}_{\boldsymbol{i}}^{+}$ 
and $\boldsymbol{p}_{\boldsymbol{i}}^{-}$) from the sampled points (in analogy of $\boldsymbol{p}_{\boldsymbol{i}}$ on the insulator) in the Parallel-Plate Capacitor model. In other words, once the locations of positive and negative primitives are deduced, the insulator between them can be located as the zero set of implicit function shown in Eq. (2) correspondingly.

\section{$3 \quad$ Field Fitting}

In this section, we introduce our Field Fitting approach by the analogy between surface reconstruction and the primitives' distributions estimation of ParallelPlate Capacitor model.

Given an original 3D scattered point cloud, which can be treated as a coarse discrete representation of the insulator, we can estimate the initial locations of the positive/negative primitives with the guidance of the Parallel-Plate Capacitor model. Then the surface fitting can be carried out by reconstructing of continuous insulator based on the estimated location of primitives.

\subsection{The Analogy of Parallel-Plate Capacitor Model}

As mentioned before, we use $P=\left\{\boldsymbol{p}_{\boldsymbol{i}}\right\}$ to denote points sampled from the original surface (the insulator), and $N=\left\{\boldsymbol{n}_{\boldsymbol{i}}\right\}$ are the corresponding normal values. The normal values can be captured by $3 \mathrm{D}$ scanner or computed by methods like [13, in our approach, the normals of input points are precalculated by the method proposed in 13 .

Similar to the off-set points strategy, we can infer the off-set points $P^{+}=$ $\left\{\boldsymbol{p}_{\boldsymbol{i}}^{+}\right\}$and $P^{-}=\left\{\boldsymbol{p}_{\boldsymbol{i}}^{-}\right\}$shown in Figure $2(\mathrm{a})$. With the analogy of the ParallelPlate Capacitor, $P^{+}$and $P^{-}$are regarded as subsets of positive/negative primitives of the parallel conductors. As mentioned before, We can estimate the global potential based on the distribution of the primitives on both sides of capacitor. However, the distribution of all primitives are still unknown.

Taking a positive primitive $\boldsymbol{p}_{\boldsymbol{i}}^{+}$for example, to compute the primitives' distribution in its neighborhood, we define a local coordinate whose origin is at

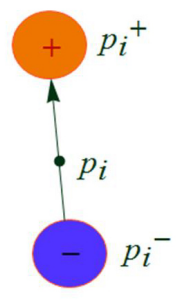

(a)

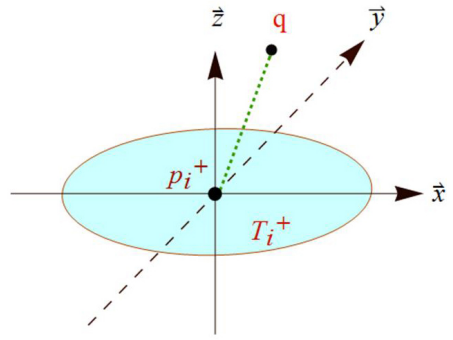

(b)

Fig. 2. From left to right, (a): given a point $\boldsymbol{p}_{i}$ on the surface, we can infer a paired primitives $\boldsymbol{p}_{\boldsymbol{i}}^{+}$and $\boldsymbol{p}_{\boldsymbol{i}}^{-}$whose middle is at $\boldsymbol{p}$; (b): the distribution of primitives near $\boldsymbol{p}_{\boldsymbol{i}}^{+}$ 
$\boldsymbol{p}_{\boldsymbol{i}}^{+}$, and direction of local $z$ axis is the same as $\boldsymbol{n}_{\boldsymbol{i}}$, as shown in Figure 2(b). $T_{i}^{+}$is the tangent plane corresponding to $\boldsymbol{p}_{\boldsymbol{i}}^{+} . N\left(\boldsymbol{p}_{\boldsymbol{i}}^{+}\right)$includes $\boldsymbol{p}_{\boldsymbol{i}}^{+}$'s neighboring primitives in $P^{+}$. Thus the distribution of primitives on the $T_{i}^{+}$in the capacitor can be described by the probability below:

$$
h(\boldsymbol{t})=\frac{1}{2 \pi|\Sigma|^{\frac{1}{2}}} \cdot e^{-\boldsymbol{t}^{T} \Sigma^{-1} \boldsymbol{t}}
$$

where $\boldsymbol{t}=\left(\begin{array}{l}x \\ y\end{array}\right)$ and $\Sigma$ is covariance of the Gaussian distribution. That is, we suppose the primitives are distributed on a local tangent plane and $h(\boldsymbol{t})$ defines the probability of those primitives in local tangent plane whose distance from $\boldsymbol{p}_{\boldsymbol{i}}^{+}$ are $\boldsymbol{t}$. The covariance $\Sigma$ can be estimated by the neighboring primitives $N\left(\boldsymbol{p}_{\boldsymbol{i}}^{+}\right)$ as below:

$$
\Sigma=\frac{1}{k} \sum^{j}\left(\left(\boldsymbol{x}_{\boldsymbol{j}}-\boldsymbol{p}_{\boldsymbol{i}}^{+}\right) \perp \boldsymbol{T}_{\boldsymbol{i}}^{+}\right) \cdot\left(\left(\boldsymbol{x}_{\boldsymbol{j}}-\boldsymbol{p}_{\boldsymbol{i}}^{+}\right) \perp \boldsymbol{T}_{\boldsymbol{i}}^{+}\right)^{T}
$$

where $k=\left|N\left(\boldsymbol{p}_{\boldsymbol{i}}^{+}\right)\right|, \boldsymbol{x}_{\boldsymbol{j}} \in N\left(\boldsymbol{p}_{\boldsymbol{i}}^{+}\right)$and $\perp$ denote the projection operator. Eq. (4) means that $\Sigma$ can be achieved by the neighboring primitives' projection points on tangent plane. Note that $\Sigma$ can be decomposed through Singular Value Decomposition (SVD) as below:

$$
\Sigma=[\boldsymbol{u} \boldsymbol{v}]\left[\begin{array}{cc}
a^{2} & 0 \\
0 & b^{2}
\end{array}\right]\left[\begin{array}{l}
\boldsymbol{u}^{T} \\
\boldsymbol{v}^{T}
\end{array}\right]
$$

Then the expected local potential at location $\boldsymbol{q}$ yielded by primitives on plane $T_{i}^{+}$can be computed as:

$$
W_{T_{i}^{+}}(\boldsymbol{q})=\int_{-\infty}^{+\infty} \int_{-\infty}^{+\infty} h(\boldsymbol{t}) \cdot w_{i}^{+}(\boldsymbol{r}) d x d y
$$

where $\boldsymbol{t}=\left(\begin{array}{l}x \\ y\end{array}\right), \boldsymbol{r}=\boldsymbol{q}-\left(\begin{array}{l}x \\ y \\ 0\end{array}\right)$ and $w_{i}^{+}(\boldsymbol{r})$ is the potential function for one positive primitive in $T_{i}^{+}$, in our approach we express $w_{i}^{+}(\boldsymbol{r})$ as a Gaussian function due to its natural smooth blending characteristics. In other words, $w_{i}^{+}(\boldsymbol{r})=e^{-\frac{|\boldsymbol{r}|^{2}}{2 m^{2}}}$. Eq. (6) means the local potential value around $\boldsymbol{p}_{\boldsymbol{i}}^{+}$is yielded by all the primitives on its tangent plane $T_{i}^{+}$with probability $h(\boldsymbol{t})$.

Substituting Eq. (3) into Eq. (6), we have:

$$
\begin{gathered}
W_{T_{i}^{+}}(\boldsymbol{q})=C_{i}^{+} \cdot e^{-\frac{1}{2}\left(\boldsymbol{q}^{{ }^{T}} \cdot \Sigma_{g}^{-1} \cdot \boldsymbol{q}^{\prime}\right)} \\
C_{i}^{+}=\frac{2 \pi}{\sqrt{\frac{1}{a^{2}}+\frac{1}{m^{2}}} \sqrt{\frac{1}{b^{2}}+\frac{1}{m^{2}}}}
\end{gathered}
$$




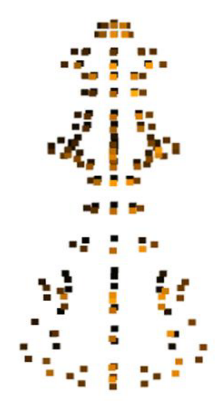

(a)

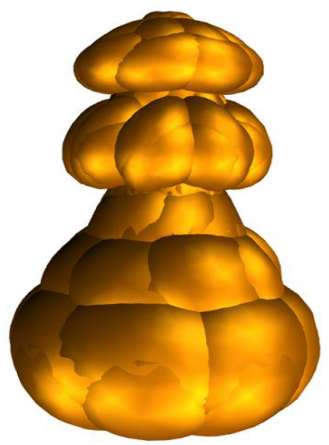

(b)

Fig. 3. From left to right, (a): the original point cloud sampled from a chess; (b): the ellipsoids which stand for the support regions of all $W_{T_{i}^{+}} / W_{T_{i}^{-}}$

$$
\Sigma_{g}=\left[\begin{array}{ccc}
a^{2}+m^{2} & 0 & 0 \\
0 & b^{2}+m^{2} & 0 \\
0 & 0 & m^{2}
\end{array}\right]
$$

where $q^{\prime}$ is the local coordinate of $q$ corresponding to point $\boldsymbol{p}_{\boldsymbol{i}}^{+}$and

$$
\boldsymbol{q}^{\prime}=\left(\boldsymbol{u}, \boldsymbol{v}, \boldsymbol{n}_{\boldsymbol{i}}\right)^{T} \cdot\left(\boldsymbol{q}-\boldsymbol{p}_{\boldsymbol{i}}^{+}\right)
$$

Note that the local potential function $W_{T_{i}^{+}}$in Eq. (7) is a Gaussian function whose covariance is $\Sigma_{g}$. As Gaussian function is a compact support function, whose support region is an ellipsoid defined by $e^{-\frac{1}{2}\left(\boldsymbol{q}^{T^{T}} \cdot \Sigma_{g} \cdot \boldsymbol{q}^{\prime}\right)} \leq d$ min where dmin is the minimal value according to the computer's precision limitation. Figure 3(b) shows the support regions of all $W_{T_{i}^{+}}$and $W_{T_{i}^{-}}$for a $3 \mathrm{D}$ chess model. The parameter $m$ in Eq. (7) controls the thickness of support regions along the direction of $n_{i}$ (shaped like a cake) in Figure 3(b), in our approach, the parameter $m$ in $W_{T_{i}^{+}}$is estimated by:

$$
m=\sqrt{\frac{1}{k} \sum_{\boldsymbol{x} \in N\left(\boldsymbol{p}_{\boldsymbol{i}}^{+}\right)}\left(\left(\boldsymbol{x}-\boldsymbol{p}_{\boldsymbol{i}}^{+}\right) \cdot \boldsymbol{n}_{\boldsymbol{i}}\right)^{2}}
$$

where $k=\left|N\left(\boldsymbol{p}_{\boldsymbol{i}}^{+}\right)\right|$is the number of neighboring positive primitive of $\boldsymbol{p}_{\boldsymbol{i}}^{+}$. Eq. (9) means that we get the thickness of the support regions along the direction of $\boldsymbol{n}_{i}$ through stat the variance of neighboring primitives' projection points on $\boldsymbol{p}_{\boldsymbol{i}}^{+}$'s normal direction $\boldsymbol{n}_{\boldsymbol{i}}$. And it's the same for negative primitives.

\subsection{Field Fitting Model Construction}

As we explained in section 2.1, the zero equipotential surface does not necessarily appear in the middle of the capacitor(the insulator) which goes through $P=\left\{\boldsymbol{p}_{\boldsymbol{i}}\right\}$. The positions of positive/negative primitives need to be adjusted to fit the insulator. And in our approach, moving tangent planes $T_{i}^{+} / T_{i}^{-}$are 
equivalent to moving primitives near $p_{i}^{+} / p_{i}^{-}$(because it is supposed that the neighboring primitives of $p_{i}^{+} / p_{i}^{-}$are distributed in $T_{i}^{+} / T_{i}^{-}$with some probabilities in section 2.1).

Therefore, the local potential function (Eq. (7)) is reformulate as:

$$
W_{T_{i}^{+}}\left(d_{i}, \boldsymbol{q}\right)=C_{i}^{+} \cdot e^{-\frac{1}{2}\left(\boldsymbol{q}^{\prime \prime T} \cdot \Sigma_{g}^{-1} \cdot \boldsymbol{q}^{\prime \prime}\right)}
$$

where $\boldsymbol{q}^{\prime \prime}=\left(\boldsymbol{u}, \boldsymbol{v}, \boldsymbol{n}_{\boldsymbol{i}}\right)^{T} \cdot\left(\boldsymbol{q}-\left(\boldsymbol{p}_{\boldsymbol{i}}^{+}+d_{i} \cdot n_{i}\right)\right)$. Note that this is the same for negative primitives.

The global potential at $q$ can be inferred to be a combination of all local potentials:

$$
F(D, \boldsymbol{q})=\sum_{i} W_{T_{i}^{+}}\left(d_{i}, \boldsymbol{q}\right)+\sum_{i} W_{T_{i}^{-}}\left(d_{i}, \boldsymbol{q}\right)
$$

where $F(D, \boldsymbol{q})$ denotes the global potential value after doing movement $D=$ $\left\{d_{i}\right\} . W_{T_{i}^{+}}\left(d_{i}, \boldsymbol{q}\right)$ and $W_{T_{i}^{-}}\left(d_{i}, \boldsymbol{q}\right)$ compute the local potential function after doing $d_{i}$ movement respectively.

Given an ideal local tangent plane movement set $D_{0}=\left\{d_{i 0}\right\}$, we have a zero potential surface defined by $F\left(D_{0}, \boldsymbol{p}_{\boldsymbol{i}}\right)=0$. Therefore, the energy function $J$ measuring the distance between the potential function $F$ and the exact surface can be defined as below:

$$
J(D)=\sum_{\boldsymbol{p}_{i} \in P} F\left(D, \boldsymbol{p}_{\boldsymbol{i}}\right)^{2}-\sum_{\boldsymbol{p}_{\boldsymbol{i}} \in P} F\left(D_{0}, \boldsymbol{p}_{\boldsymbol{i}}\right)^{2}=\sum_{\boldsymbol{p}_{\boldsymbol{i}} \in P} F\left(D, \boldsymbol{p}_{\boldsymbol{i}}\right)^{2}
$$

From the definition of $J$, it is easy to infer that all we need is to find $D_{0}=\left\{d_{i 0}\right\}$, which can generate the minimum energy $J$.

However, solving the minimization of Eq. (12) with conventional Broyden's method 12 is too slow to be acceptable. In our approach, we introduce a greedy method which is more efficient according to empirical studies. The greedy method estimates each $d_{i}$ through a local potential function. For each paired plane $T_{i}^{+}$and $T_{i}^{-}$, we use $S_{p i}\left(S_{p i} \subset P\right)$ to denote the points which are in the support region of $W_{T_{i}^{+}}$or $W_{T_{i}^{-}}$. Then the local energy function $J_{i}$ can be rewrited as:

$$
J_{i}\left(d_{i}\right)=\sum_{\boldsymbol{x} \in S_{p_{i}}} F(D, \boldsymbol{x})^{2}=\sum_{\boldsymbol{x} \in S_{p_{i}}}(A(\boldsymbol{x})+B(\boldsymbol{x}))^{2}
$$

where

$$
\begin{gathered}
A(\boldsymbol{x})=\sum_{\boldsymbol{T}_{j}^{+} \in R_{i}^{+}} W_{\boldsymbol{T}_{j}^{+}}\left(d_{j}, \boldsymbol{x}\right)+\sum_{\boldsymbol{T}_{j}^{-} \in R_{i}^{-}} W_{\boldsymbol{T}_{j}^{-}}\left(d_{j}, \boldsymbol{x}\right) \\
B(\boldsymbol{x})=W_{T_{i}^{+}}\left(d_{i}, \boldsymbol{x}\right)+W_{T_{i}^{-}}\left(d_{i}, \boldsymbol{x}\right)
\end{gathered}
$$

where $R_{i}^{+}=T^{+}-\left\{\boldsymbol{T}_{\boldsymbol{i}}^{+}\right\}, R_{i}^{-}=T^{-}-\left\{\boldsymbol{T}_{\boldsymbol{i}}^{-}\right\}$, so $A(\boldsymbol{x})$ is the potential yield by primitives other than $W_{T_{i}^{+}}$, and $B(\boldsymbol{x})$ denote the potential yield by $W_{T_{i}^{+}}$. Then $d_{i 0}$ can be achieved by solving:

$$
d_{i 0}=\underset{d_{i}}{\arg \min } J_{i}\left(d_{i}\right)
$$


Table 1. Algorithm to obtain the potential function

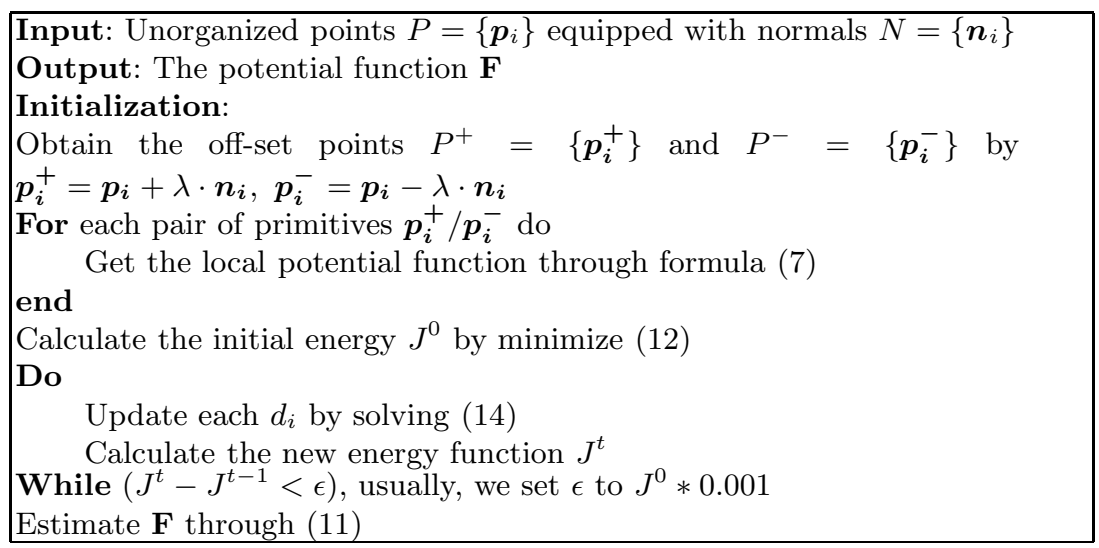

Note that we split the energy function into two parts, the part of $A(\boldsymbol{x})$ is a constant value independent of $d_{i}$, and $B(\boldsymbol{x})$ is dependent of $d_{i}$. Therefore solving Eq. (14) with Quasi-Newton method [12] is much simpler than that of Eq. (12). Table 1 lists the proposed algorithm to obtain the potential function $F$.

\subsection{Multi-scaling Strategy}

Due to inevitable scanning errors, the input point cloud often has some unsampled regions which introduce holes on the surface. To fix these holes, we utilize the multi-scaling strategy in our Field fitting method which is widely used by the previous surface reconstruction approaches [10 14 15].

Point cloud thinning method [15] is employed to sample the multi-resolution point clouds from $P$, which down samples the point cloud through picking out the points with the minimum distance to their nearest neighbor. For each level of point cloud $L^{i}$, we can use the algorithm described in table 1 to estimate its corresponding $i$ th-level global potential function $F^{i}$, and the overall global potential function $\mathrm{F}$ can be simply expressed as the sum of $F^{i}$. In our approach, we only use a very small number of levels, usually 2 or 3 and the down sampling ratio is set to be 0.1 for adjacent level. We apply Field Fitting to a incomplete bunny model (Figure 4 (a)), the reconstruction result by 1 level Field Fitting is shown in Figure 4(b), and the reconstruction result by multi levels (2 levels) Field Fitting is shown in Figure 4(c), it is noticeable that multi-scaling strategy can effectively fix holes which are introduced by unsampled regions.

\section{Visualization}

Polygonization [16, ray tracing [17] as well as point based rendering [9] can be used to visualize the surface fitting result. We employ the point based rendering method in our approach. 


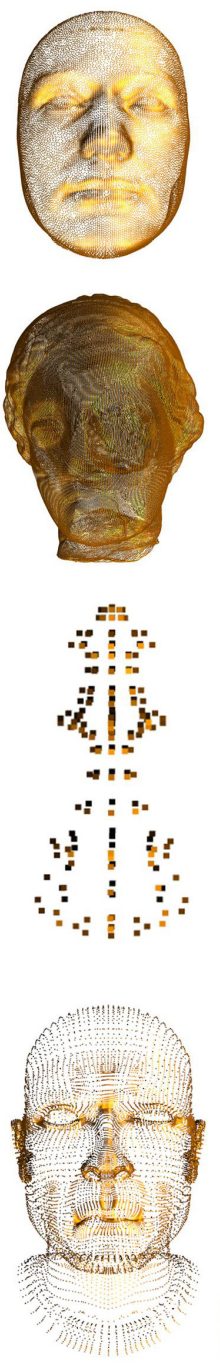

(a)
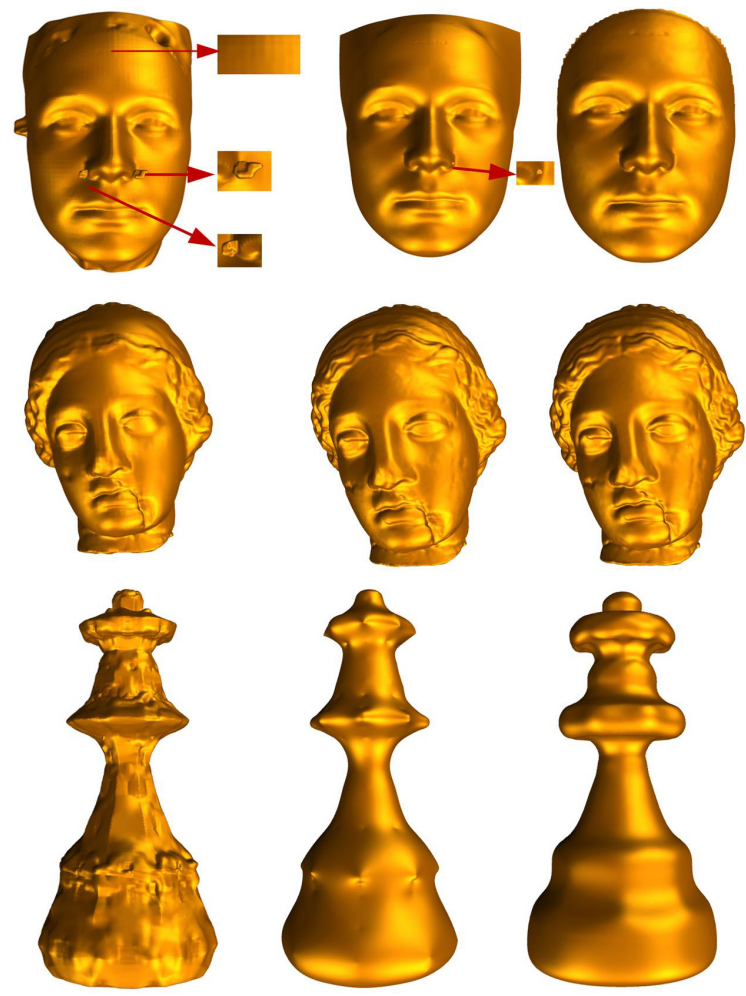

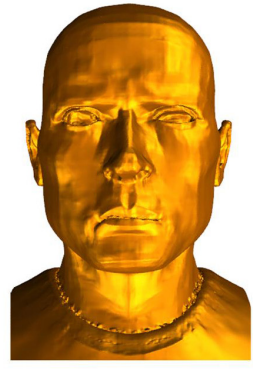

(b)

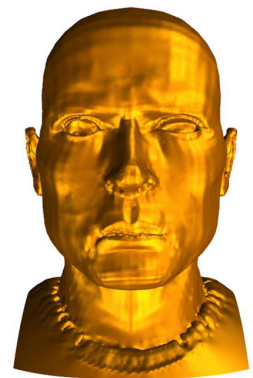

(c)

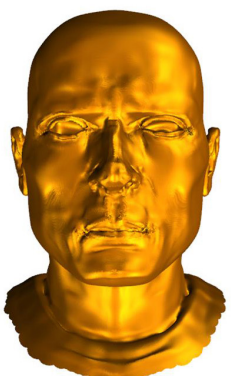

(d)

Fig. 4. The testing models and the reconstruction results. From left to right, the original models; MPU reconstruction results; Fast RBF reconstruction results; Field Fitting reconstruction results. From top to bottom, frontal face model (33,053 points), 'igea' (134,345 points), chess model (186 points), head model (15,941 points).

After obtaining the global potential function, a tracking process is carried out to obtain the zero level set points of the global potential function. This tracking process is very similar to the implicit surface polygonizing process [16], the only difference is that we do not polygonize the tracked cubes which are intersected with implicit surface, but only mark the centers of these cubes as the coarse zero level set points and by using binary search, a precisely locations the zero level set points can be found. 
Table 2. Time, memory and triangles/points generated by different method, note that MPU and fRBF(denote Fast RBF) approach generate triangles while Our Field Fitting approach generate points

\begin{tabular}{|l|r|r|r|r|r|}
\hline \multirow{2}{*}{ Model } & Approach & $\begin{array}{l}\text { Fitting } \\
\text { time }\end{array}$ & $\begin{array}{l}\text { Isosurface } \\
\text { extraction }\end{array}$ & Memory & Tris/Points \\
\hline \multirow{3}{*}{$\begin{array}{l}\text { Frontal } \\
\text { Face }\end{array}$} & MPU & $1.1 \mathrm{~s}$ & $20.2 \mathrm{~s}$ & $325 \mathrm{M}$ & $1,603 \mathrm{~K}$ \\
\cline { 2 - 6 } & fRBF & $203.4 \mathrm{~s}$ & $123.9 \mathrm{~s}$ & $105 \mathrm{M}$ & $643 \mathrm{~K}$ \\
\cline { 2 - 6 } Igea & Ours & $36.6 \mathrm{~s}$ & $183.3 \mathrm{~s}$ & $850 \mathrm{M}$ & $1,037 \mathrm{~K}$ \\
\cline { 2 - 6 } & MPU & $4.6 \mathrm{~s}$ & $20.1 \mathrm{~s}$ & $320 \mathrm{M}$ & $1,253 \mathrm{~K}$ \\
\cline { 2 - 6 } & ORBF & $105.6 \mathrm{~s}$ & $396.3 \mathrm{~s}$ & $162 \mathrm{M}$ & $1,042 \mathrm{~K}$ \\
\hline \multirow{3}{*}{ Chess } & Ours & $141.1 \mathrm{~s}$ & $297.6 \mathrm{~s}$ & $1500 \mathrm{M}$ & $2,128 \mathrm{~K}$ \\
\cline { 2 - 6 } & fRBF & $0.23 \mathrm{~s}$ & $22.1 \mathrm{~s}$ & $180 \mathrm{M}$ & $2,092 \mathrm{~K}$ \\
\cline { 2 - 6 } & Ours & $0.14 \mathrm{~s}$ & $7.3 \mathrm{~s}$ & $73 \mathrm{M}$ & $393 \mathrm{~K}$ \\
\hline \multirow{3}{*}{ Head } & MPU & $17.2 \mathrm{~s}$ & $28.4 \mathrm{~s}$ & $374 \mathrm{M}$ & $1,929 \mathrm{~K}$ \\
\cline { 2 - 6 } & fRBF & $1051.6 \mathrm{~s}$ & $396.0 \mathrm{~s}$ & $460 \mathrm{M}$ & $910 \mathrm{~K}$ \\
\cline { 2 - 6 } & Ours & $17.4 \mathrm{~s}$ & $199.0 \mathrm{~s}$ & $720 \mathrm{M}$ & $1,649 \mathrm{~K}$ \\
\hline
\end{tabular}

\section{Experiments}

The experiments are performed on an Intel Core2 Q6600 with 2GBytes ram running windows server 2003. We make comparison between the proposed approach and two representative existing methods, namely, MPU[10], and FastRBF [5], to perform evaluation.

We firstly test a frontal face which has 33053 points, as shown in the first row of Figure 4. It is noticeable that there are some extra artifacts near the nose in the result by MPU because the inherent limitation of the quadric function for local domain operation and there are also artifacts near nose in RBF's result. Moreover, the narrow scar on the forehead is smoothed off by MPU due to the quadric function's strong smoothing effect. RBF can produce more reasonable and competitive result (first row third column). But some subtle details in the face are missed. Fortunately, it is noticeable that more subtle details (the scar on forehead) are preserved naturally in result of Field Fitting(the forth column) than that of MPU and RBF, i.e. in the result of our Field Fitting approach, the scar on forehead is clearly to be seen, but in MPU's result, the scar is smooth off and in RBF's result the scar is not so clearly as ours. Further, for a head model in forth row, there are many strange stripes in the result of RBF and MPU, Field Fitting outperforms RBF and MPU by giving natural and realistic surface reconstruction with better global stability and local details' preservation.

We also perform test on the model with very sparse point cloud. As shown in third row of Figure 4, neither MPU nor RBF produces qualified result because of some unnatural distortion and artifacts. The proposed Field Fitting method works well on this sparse model (forth column). This demonstrates that the proposed method outperforms both the two previous methods for its higher robustness. 
We also compare the time consumption, memory and triangles/points generated by different methods in Table2. Although in this table, our approach is neither fastest nor most memory saving (due to the deep space splits for isosurface extraction), it is noticeable that our approach generate a smooth and detail reserved surfaces which have nicer appearance than MPU and FastRBF.

\section{Conclusion}

In this paper, we present a novel implicit surface reconstruction approach by an analogy of classic physical polar field model. The experimental results show that the proposed Field Fitting approach outperforms the previous methods by generating results with more subtle details while keeping global smoothness. Moreover, it is noticeable that the proposed approach keeps robust to sparse input point cloud.

Though the proposed method provides a better solution on implicit surface fitting, we notice that there are still several problems with it. For example, the proposed approach needs larger memory to extract isosurfaces than the representative existing approaches. And, the efficiency still needs improved. In our future work, we will pay more attention to the reduction of memory requirement and try to explore a more efficient fitting strategy to strengthen the method's usability.

Acknowledgments. This paper is supported by National Science Foundation of China (Grant No. 60873124), and thanks to FarField Technology Ltd who provide us a demonstration license of FastRBF Toolkit with a capability of 1000k input points.

\section{References}

1. Bloomenthal, J., Bajaj, C.: Introduction to Implicit Surfaces. Morgan Kaufmann, San Francisco (1997)

2. Dierckx, P.: Curve and Surface Fitting with Splines. Oxford University Press, Oxford (1993)

3. Floater, M., Hormann, K.: Surface Parameterization: a Tutorial and Survey. Advances In Multiresolution For Geometric Modelling (2005)

4. Blane, M., Lei, Z., Çivi, H., Cooper, D.: The 3L Algorithm for Fitting Implicit Polynomial Curves and Surfaces to Data. IEEE Transactions on Pattern Analysis and Machine Intelligence, 298-313 (2000)

5. Carr, J., Beatson, R., Cherrie, J., Mitchell, T., Fright, W., McCallum, B., Evans, T.: Reconstruction and representation of $3 \mathrm{D}$ objects with radial basis functions. In: Proceedings of the 28th annual conference on Computer graphics and interactive techniques, pp. 67-76 (2001)

6. Carr, J., Beatson, R., McCallum, B., Fright, W., McLennan, T., Mitchell, T.: Smooth surface reconstruction from noisy range data. ACM GRAPHITE 3, 119126 (2003)

7. Hoppe, H., DeRose, T., Duchamp, T., McDonald, J., Stuetzle, W.: Surface reconstruction from unorganized points. In: Proceedings of the 19th annual conference on Computer graphics and interactive techniques, pp. 71-78 (1992) 
8. Alexa, M., Behr, J., Cohen-Or, D., Fleishman, S., Levin, D., Silva, C.: Point set surfaces. In: Proceedings of the conference on Visualization 2001, pp. 21-28 (2001)

9. Alexa, M., Behr, J., Cohen-Or, D., Fleishman, S., Levin, D., Silva, C.T.: Computing and Rendering Point Set Surfaces. Computing 9(1), 3-15 (2003)

10. Ohtake, Y., Belyaev, A., Alexa, M., Turk, G., Seidel, H.: Multi-level partition of unity implicits. In: International Conference on Computer Graphics and Interactive Techniques, pp. 463-470 (2003)

11. Kazhdan, M., Bolitho, M., Hoppe, H.: Poisson Surface Reconstruction. Indicator 1(1) (2006)

12. Press, W., Teukolsky, S., Vetterling, W., Flannery, B.: Numerical Recipes in C++. Cambridge Univ. Press, Cambridge (2002)

13. Mitra, N., Nguyen, A.: Estimating surface normals in noisy point cloud data. In: Proceedings of the nineteenth annual symposium on Computational geometry, pp. 322-328 (2003)

14. Ohtake, Y., Belyaev, A., Seidel, H.: A multi-scale approach to 3D scattered data interpolation with compactly supported basis functions. In: Shape Modeling International 2003, pp. 153-161 (2003)

15. Tobor, I., Reuter, P., Schlick, C.: Multi-scale reconstruction of implicit surfaces with attributes from large unorganized point sets. In: Proceedings of Shape Modeling Applications, pp. 19-30 (2004)

16. Bloomenthal, J.: An implicit surface polygonizer. Graphics Gems IV 349 (1994)

17. Hart, J.: Sphere tracing: a geometric method for the antialiased ray tracing of implicit surfaces. The Visual Computer 12(10), 527-545 (1996) 